\title{
МЕТОДИКА ЦИФРОВОЙ ОБРАБОТКИ СИГНАЛОВ ДЛЯ ПОВЬШЕНИЯ РАЗРЕШАЮЩЕЙ СПОСОБНОСТИ ГАЗОСИГНАЛИЗАТОРА
}

\section{DIGITAL SIGNAL PROCESSING \\ TECHNIQUE FOR INCREASED \\ RESOLUTION OF A GAS DETECTOR}

\section{E. Shchuchkin}

Summary. The study solves the problem of increasing resolution ability of gas detectors with the use of methods of digital signal processing of the signal from an analog gas sensor. The task is significant for industrial and domestic gas detectors. The article provides a diagram of the digital processing path for a typical gas detector module based on a microcontroller with an analog-to-digital converter, an operational amplifier and a gas sensor. The proposed signal-processing path includes temperature compensation unit and a digital low-pass filter. The paper proposes a quasi-linear model of the dependence of the gas sensor signal of temperature for generating of correction coefficients that can compensate the temperature error when operating in an extended temperature range. An algorithm proposed for the implementation of a digital low-frequency filter that increases the resolution of the gas detector based on interpolating the initial discrete data with cubic splines. An important mathematical result of the study is that the method of selecting interpolation nodes allows minimizing the absolute error modulus of the approximating curve with respect to the initial data. Based on the simulation results, a comparison made be-tween the filter described in the article and the median filter. From the point of view of the theoretical study, the proposed method of digital signal processing can increase the resolution of gas detectors in determining the gas concentration, allowing abandoning installation of precision components and reducing the cost of the final product.

Keywords: digital signal processing, analog sensor, gas detector, median filter, interpolation, sensor resolution.

\author{
Щучкин Евгений Юрьевич \\ Аспирант, Национальный исследовательский \\ университет «Московский институт электронной \\ техники» \\ shchuchkin-pkims@yandex.ru
}

Аннотация. В работе решается задача повышения разрешающей способности газосигнализаторов за счет применения методов цифровой обработки сигнала от аналогового датчика газа. Задача является значимой для газосигнализаторов промышленного и бытового назначения. В статье приведена схема тракта цифровой обработки для типового устройства газосигнализатора, выполненного на основе микроконтроллера с аналого-цифровым преобразователем, операционного усилителя и датчика газ. Предложенный тракт обработки сигнала включает в себя блоки компенсации влияния температуры и фильтр низких частот. В работе предложена квазилинейная модель зависимости сигнала датчика газа от температуры для формирования поправочных коэффициентов, компенсирующих ошибку при работе в расширенном диапазоне температур. Предложен алгоритм реализации цифрового фильтра низких частот, повышающего разрешающую способность газосигнализатора методом интерполяции исходных отчетов кубическими сплайнами. Важный математический результат проведенного исследования состоит в том, что методика выбора узлов интерполяции позволяет минимизировать модуль абсолютной ошибки аппроксимирующей кривой по отношению к исходным данным. По результатам моделирования было проведено сравнение описанного в статье фильтра со медианным фильтром. С точки зрения проведенного теоретического исследования можно заключить, что предложенный метод цифровой обработки сигналов может повысить разрешающую способность газосигнализаторов при определении концентрации газа, позволяя отказаться от прецизионных компонентов и снизить стоимость конечного изделия.

Ключевые слова: цифровая обработка сигналов, аналоговый датчик, газосигнализатор, медианный фильтр, интерполяция, разрешающая способность.

Одним из широко распространенных типов датчиков газа является электрохимический датчик, поскольку он обладает сравнительно низкой стоимостью, линейной зависимостью напряжения от концентрации газа, низким энергопотреблением и высокой избирательностью [1]. Как правило, выходной сигнал с таких датчиков усиливается операционным усилителем и подается на вход аналого-цифрового преобразователя (АЦП). Оцифрованный сигнал обрабатывается микроконтроллером, установленным на модуль. Микроконтроллер выполняет первичную обработку сигнала, вычисляет текущее значение концентрации газа в окружающей среде и передает данные на централизованную систему управле- 


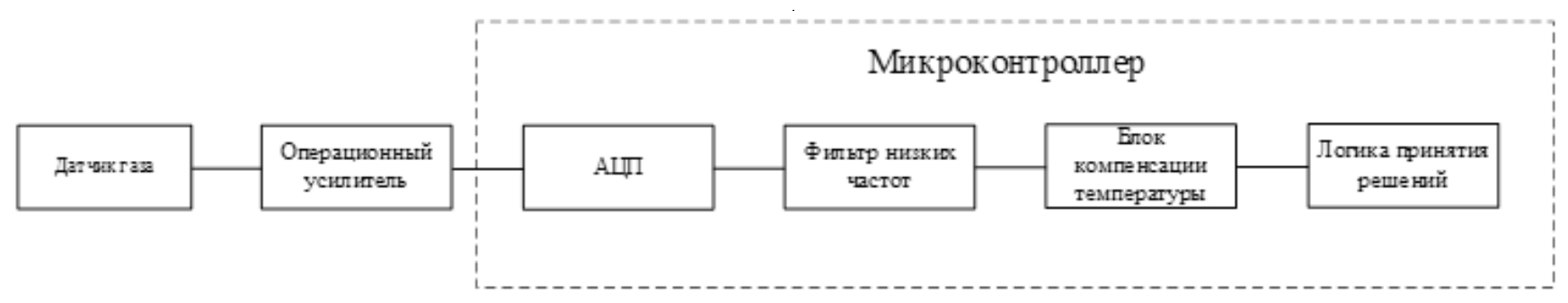

Рис. 1. Тракт цифровой обработки сигнала датчика газа, обеспечивающий повышенную разрешающую способность

ния или самостоятельно принимает решение о выдаче аварийных сигналов [2].

Для снижения конечной стоимости изделия разработчикам приходится применять дешевые компоненты с высоким разбросом характеристик, что в свою очередь приводит к повышению уровня шумов, температурной нестабильности и, как следствие, к увеличению ошибки при определении уровня концентрации газа. Одним из способов борьбы с данными эффектами является цифровая обработка сигналов (ЦОС).

При цифровой фильтрации исходных отчетов выборки АЦП зачастую применяется медианный фильтр в виду простоты его реализации $[3,4]$. Суть метода заключается в сортировке по возрастанию $N$ исходных отчетов с их последующей заменой значением отчета, оказавшегося в середине упорядоченного списка. В случае с четным количеством исходных отчетов на выход фильтра выдается среднее арифметическое двух центральных отчетов упорядоченного списка. Медианный фильтр позволяет сгладить выбросы в исходном сигнале и провести децимацию отчетов $[5,6]$.

Тем не менее, данный способ цифровой фильтрации обладает рядом недостатков. На выходе медианного фильтра сигнал остается дискретным, а значит обладает шумом квантования. Фильтр не подразумевает преобразования исходных дискретных данных с шумами в непрерывный сигнал за счет усреднения с целью повышения разрешающий способности, т.е. так называемый дизеринг [7].

Разрешающая способность газосигнализатора может быть повышена если заменить дискретный сигнал непрерывной кусочно-гладкой функцией. Данная задача может быть решена интерполяцией кубическими сплайнами. В таком случае встает вопрос выбора узлов интерполяции. Значения на выходе медианного фильтра несут случайный характер и могут не коррелировать с соседними отчетами в окне. В данной статье описана методика получения узлов интерполяционной сетки для построения зависимости напряжения от концентрации газа при помощи кубических сплайн-функций, а также предложена схема обработки сигнала датчика с целью повышения разрешающей способности.

\section{Постановка заАачи}

Современные газосигнализирующие устройства представляют из себя встраиваемую микропроцессорную систему, состоящую из датчика газа, датчика температуры, усилителей и микроконтроллера с АЦП. Одной из задач при разработке устройств такого класса является цифровая обработка поступающих с датчика данных. Целью цифровой обработки является фильтрация шумов, повышение разрешающей способности устройства и компенсация влияния внешних факторов, таких как изменение температуры и влажности. Тракт обработки сигнала от датчика схематично представлен на рис. 1. Задача по обеспечению высокой разрешающей способности, при снижении требований к характеристикам компонентов на плате, решается применением более сложных алгоритмов ЦОС $[8,9]$. Таким образом, требуется разработать методику цифровой обработки сигнала датчика газа с повышенной разрешающей способностью.

\section{Метолика шифровой обработки вХOАНОГО сИгна^а}

Блок компенсации температурного дрейфа на рис. 1 должен отдельно учитывать дрейф напряжения смещения операционного усилителя и дрейф сигнала датчика. Для операционного усилителя зависимость напряжения от температуры является линейной, а коэффициент пропорциональности, как правило, приведен в документации на конкретную модель усилителя. Для датчика газа зависимость является квазилинейной. Компенсация влияния температуры на сигнал датчика может быть осуществлена за счет деления входной последовательности данных на поправочный коэффициент из массива в постоянной памяти микроконтроллера в зависимости от температуры окружающей среды. Значения для мас- 


$$
k_{T}(T)=\left\{\begin{array}{c}
\frac{\frac{V(-10)}{V(25)}-\frac{V(-20)}{V(25)}}{-10+20} \cdot(T+20)+\frac{V(-20)}{V(25)}, \text { если }-20 \leq T \leq-10 ; \\
\frac{\frac{V(0)}{V(25)}-\frac{V(-10)}{V(25)}}{0+10} \cdot(T+10)+\frac{V(-10)}{V(25)}, \text { если }-10 \leq T \leq 0 ; \\
\frac{\frac{V(10)}{V(25)}-\frac{V(0)}{V(25)}}{10-0} \cdot(T+0)+\frac{V(0)}{V(25)}, \text { если } 0 \leq T \leq 10 ; \\
\cdots \\
\frac{V(50)}{\frac{V(25)}{50}-\frac{V(40)}{V(25)}} \cdot(T-40)+\frac{V(0)}{V(25)}, \text { если } 40 \leq T \leq 50,
\end{array}\right.
$$

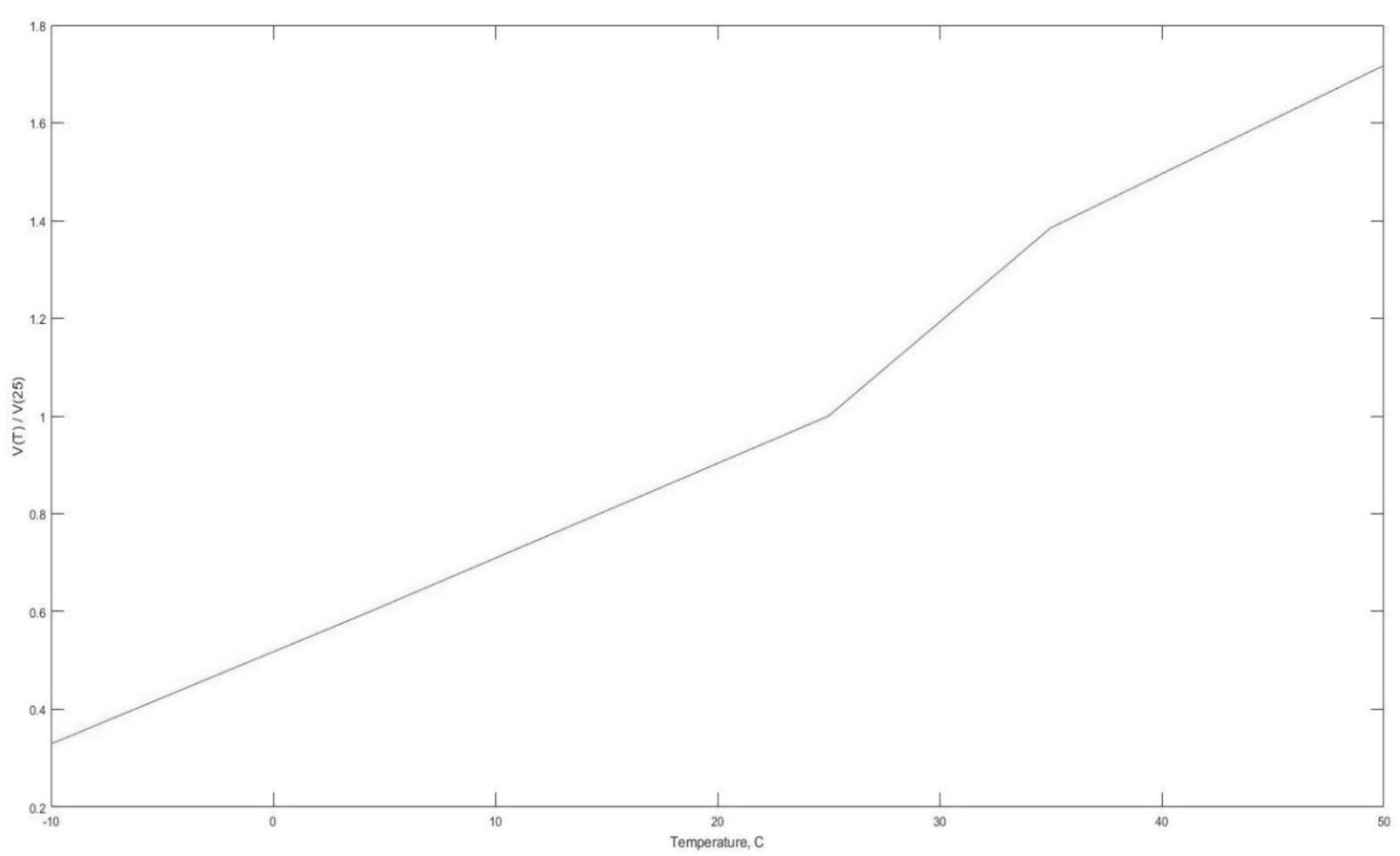

Рис. 2. Отношение напряжения на выходе датчика в зависимости от температуры к напряжению на выходе при температуре $25^{\circ} \mathrm{C}$

сива коэффициентов можно получить на основе кусочно-линейной функции $k_{T}(T)$, аппроксимирующей температурную зависимость датчика. Так при построении модели датчика газа в диапазоне температур от минус 10 до 50 градусов Цельсия с шагом в 10 градусов функция будет иметь следующий вид (формула сверху полосы), где $T$ - значение температуры окружающей среды,
$V$ - вектор значений напряжения датчика газа при заданной температуре.

Пример такой зависимости для датчика ME2-CO Winsen приведен на рис. 2. Значения напряжений для построения функции можно получить из документации на датчик или при калибровке модулей в процессе ис- 
1. function data_filtered = digital_filter(data, window, gap)

2. data_filtered = data;

3. data_length $=$ size (data);

4. data_length = data_length(2);

5. for $j=1$ : window: data_length-window

6. $\quad A=\operatorname{null}(1)$;

7. $\quad$ for $i=j: 1: j+$ window

8. $\quad A=[$ A data_filtered(i)];

$9 . \quad \operatorname{sort}(\mathrm{A})$;

$10 . \quad$ end

11. for $\mathrm{i}=\mathrm{j}: 1: \mathrm{j}+$ window

12. $\quad$ data_filtered $(\mathrm{i})=$ rms (A(gap: window-gap));

$13 . \quad$ end

14. end

15. end

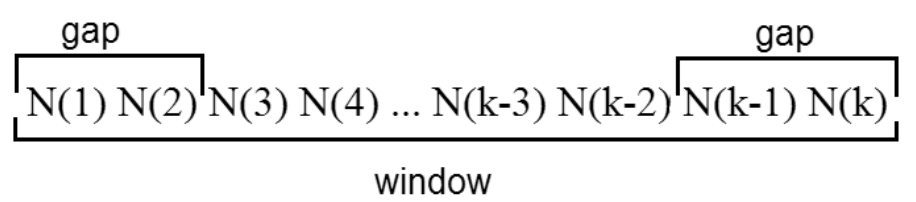

Рис. 3. Пример реализация метода для массива $N$ из $k$-элементов с параметром gap $=2$

пытаний. Данный подход применим и для компенсации влияния на измерения влажности.

Основными задачами блока фильтра низких частот на рис. 1 является децимации входной последовательности отчетов АЦП, уменьшения шумов и исключение выбросов, образованных в результате воздействия помех [10]. Поскольку данные от АЦП являются дискретными, то можно применить дизеринг и рассчитать среднее квадратичное отклонение по группе из нескольких отчетов. Таким образом, можно снизить шум квантования [11, 12]. После дизеринга сигнал останется дискретным. Для вычисления значений концентрации газа между отчетами необходимо провести интерполяцию.

Глобальные методы интерполяции обладают недостатками, связанными с высокой чувствительностью интерполяционных коэффициентов к входным данным, а также высокой степенью полинома и, как следствие, высокой вычислительной сложностью. Данных проблем можно избежать при использовании для интерполяции кубических сплайнов. Они могут быть представлены в виде полинома:

$$
P_{3}(x)=\alpha x^{3}+\beta x^{2}+\Gamma x+\delta .
$$

Кубические сплайны требуют расчета всего четырех коэффициентов и при этом не имеют разрыва производной в узлах как, например, лагранжевы сплайны [13].

\section{Методика выбора узлов интерпольчионной сетки}

Математическая задача, решаемая в работе, состоит в том, чтобы после децимации и фильтрации исходной последовательности данных определить узлы интерполяционной сетки и построить интерполяционную кривую по формуле (1), оптимизировав модуль абсолютной ошибки.

Пусть дана последовательность $N$ из $k$ дискретных отчетов с АЦП. Для получения узлов интерполяционной сетки можно воспользоваться следующим алгоритмом:

1. отсортировать отчеты $N(k)$ по возрастанию;

2. из отсортированного массива исключить gар отчетов с начала и gар отчетов с конца;

3. вычислить среднее квадратическое значение оставшихся $k-2 \cdot$ gар отчетов;

4. заменить значения исходных $k$ отчетов на среднее квадратическое значение, вычисленное на предыдущем шаге.

Параметр gар необходим чтобы исключить из входной последовательности выбросы, которые после сортировки окажутся по краям массива, а window задает размер окна фильтра. Пример разбиения исходной последовательности $k$ отчетов с окном window по предложенному методу с параметром gap $=2$ изображен на рис 3. В данном примере после применения предложенного 


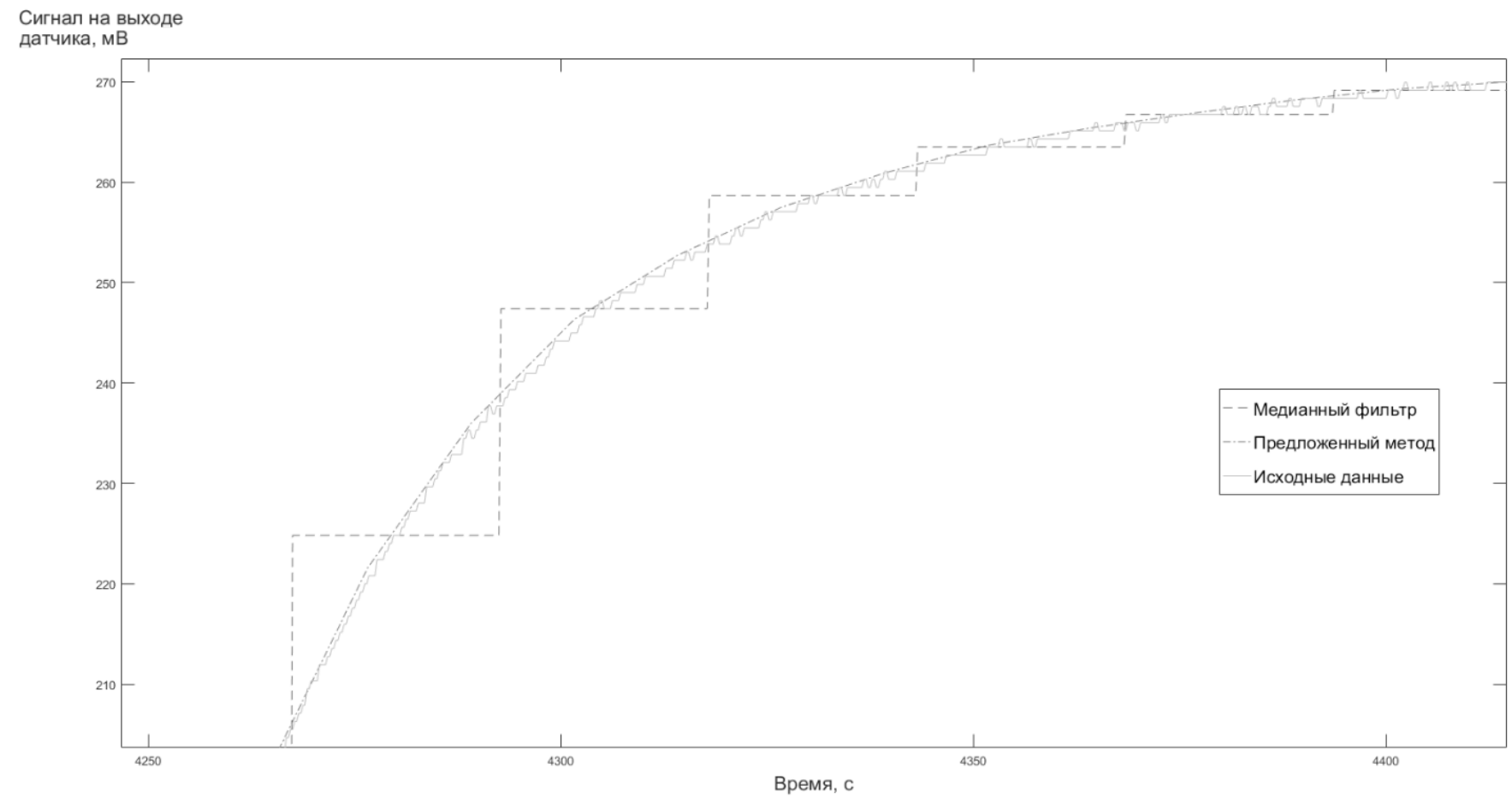

Рис. 4. Графики сигнала датчика газа без обработки, после медианного фильтра и обработанные предложенным методом

фильтра значения всех отчетов будут равны среднему квадратическому от значений отчетов

$$
\mathrm{N}(3), \mathrm{N}(4), \ldots, \mathrm{N}(\mathrm{k}-3), \mathrm{N}(\mathrm{k}-2) \text {. }
$$

Ниже представлена реализации описанного метода в виде функции на языке MATLAB. Аргументы функции: data - вектор-столбец с исходными данным, windowширина окна фильтра, gap - количество исключаемых с краев отчетов (код сверху страницы).

\section{Результаты шифровой обработки сигнала Аатчика газа}

Описанная в работе методика цифровой обработки сигнала с датчика газа была реализована в виде программы в комплексе MATLAB. В данном разделе приведены результаты фильтрации последовательности данных полученной с АЦП микроконтроллера модуля газосигнализатора СО, а также выполнено сравнение предложенной методики фильтрации с традиционным медианным фильтром. На рис. 4 приведены графики исходных данных с датчика, данные обработанные медианным фильтром и данные обработанные предложенным методом. Представленные на рис. 5 графики иллюстрируют высокую точность аппроксимации промежуточных значений при применении предложенного метода цифровой обработки.
Средствами программного комплекса MATLAB была проведена оценка быстродействия разработанного метода. В таблице 1 приведены значения времени выполнения функций фильтрации для массивов отчетов с разным количеством элементов. Можно отметить, что время выполнения программы по описанной в статье методике превышает время фильтрации медианным фильтром, но при этом составляет доли секунды. Исходя из стандарта ГОСТ Р 50759-95 время реакции систем газосигнализаторов должно составлять не более 1 минуты, а согласно EN50291-1 - не более 3 минут, что делает применимым на практике предложенный метод цифровой фильтрации [14].

\section{Зак^ючение}

В работе предложена методика цифровой обработки сигнала датчика газа, позволяющая повысить разрешающую способность газосигнализатора. Предложена схема обработки сигнала, включающая температурную компенсацию на основе построенной квазилинейной модели датчика и алгоритм реализации цифрового фильтра низких частот. Также в данной статье описана методика построения интерполирующей кривой на основе кубических сплайнов и обоснован подход к выбору интерполяционных узлов, позволяющий оптимизировать модуль абсолютной ошибки при определении НКПР и ПДК газа. 
Таблица 1. Время обработки массива данных в зависимости от количества элементов

\begin{tabular}{|l|l|l|}
$\begin{array}{l}\text { Количество элементов в } \\
\text { массиве исходных данных }\end{array}$ & $\begin{array}{l}\text { Время на обработку } \\
\text { данных медианным } \\
\text { фильтром, мс }\end{array}$ & $\begin{array}{l}\text { Время на обработку } \\
\text { данных предложенным } \\
\text { методом, мс }\end{array}$ \\
\hline 100 & 0,599 & 1,684 \\
\hline 10000 & 20,854 & 63,897 \\
\hline 50000 & 99,165 & 327,189 \\
\hline
\end{tabular}

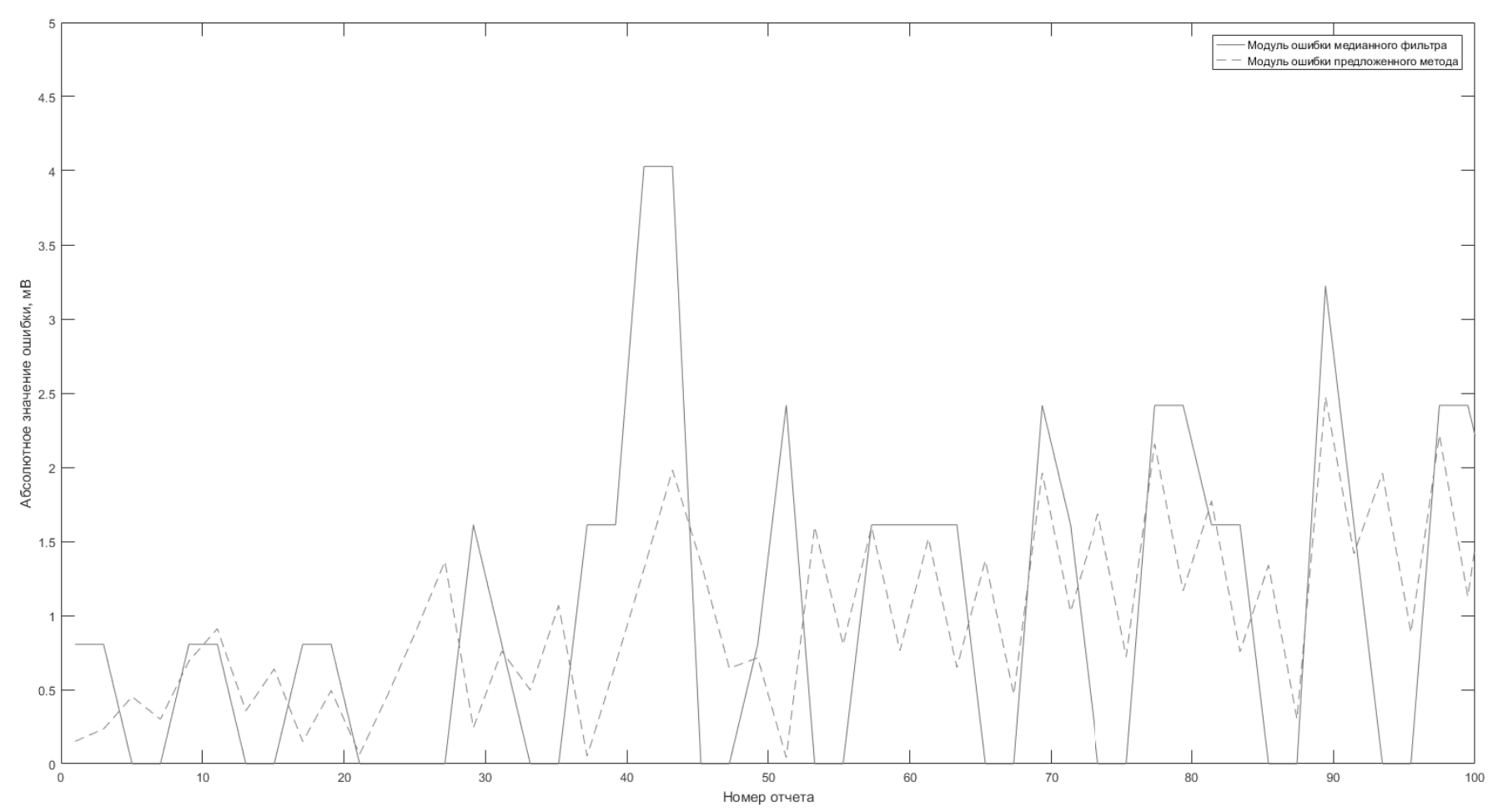

Рис. 5. Графики модуля абсолютной ошибки данных обработанных медианным фильтром и предложенным методом.

\section{ЛИТЕРАТУРА}

1. Щучкин, Е.Ю. Сравнительный анализ принципов функционирования современных датчиков газа / Е.Ю. Щучкин // Научные горизонты.- 2018.№ 4(8). - C. 229-233.

2. Камалдинов, И.А. Современное приборное обеспечение газового анализа / И А. Камалдинов, И.Н. Белоглазов, Д.С. Киреев // Записки Горного института.-2008. - T. 177.-C. 79-81.

3. Huang Ch., Sheu Yu. A high speed current-mode median filter for real-time signal processing // Asia-Pacific Conference on Circuits and Systems. - 2002. Vol.1.-P. 25-28. - DOl 10.1109/APCCAS.2002.1114901.

4. Huang Ch., Wei W., Liu B. Design of a $1.5 \mathrm{~V}$ analog current-mode median filter // Proceedings of the 2004 Intelligent Sensors, Sensor Networks and Information Processing Conference, 2004. - P. 211-215. - D0I 10.1109/ISSNIP.2004.1417464.

5. Mutauranwa L., Nleya M. An efficient median filter in a robot sensor soft ip-core // 2013 Africon. — 2013. — P. 1-5.—D0I 10.1109/AFRCON.2013.6757651.

6. Кошелев, В.И. Использование метода наименьших квадратов для повышения точности определения движения датчика угловой скорости / В.И. Кошелев, В.А. Белокуров // DSPA: Вопросы применения цифровой обработки сигналов. - 2016. - Т. 6. — № 3.— С. 550-551.

7. Садовский, А.И. Фильтрация сигналов аналоговых датчиков в системах автоматизированного электропривода / А.И. Садовский, Д.В. Бажутин // Инновационные перспективы Донбасса: материалы международной научно-практической конференции, Донецк, 20-22 мая 2015 года / ГВУЗ «Донецкий национальный технический университет» (ДонНТУ). - Донецк: Донецкий национальный технический университет, 2015. - С. 38-43.

8. Васюков, С.А. Разработка алгоритмов обработки информационного сигнала и реализация комбинированного датчика удара, наклона и движения на основе 3-осевого MEMS-акселерометра / С.А. Васюков // Радиооптика.— 2016.— № 6.—C. 47-63.— D0I 10.7463/rdopt.0616.0850702. 
9. Mainuddin F. et al. Single chip readout electronics for SAW based gas sensor systems / F. Mainuddin, U. Mittal, J. Kumar, A. Nimal, Sharma M.U. // 2017 IEEE SENSORS. - 2017. - P. 1-3.—DOI 10.1109/ICSENS.2017.8233886.

10. Федоренко, В.Ф. и др. Цифровые методы обработки данных при оценке тягового усилия тракторов / В.Ф. Федоренко, В.Е. Таркивский, Н.П. Мишуров, H.В. Трубицын // Инженерные технологии и системы. — 2021. — Т. 31.— № 1.— C. 127-142.— D0I 10.15507/2658-4123.031.202101.127-142.

11. Verma R., Ganguli R. Denoising jet engine gas path measurements using nonlinear filters // IEEE/ASME Transactions on Mechatronics. - August 2005. — Vol. 10, No. 4. - P. 461-464.—DOI 10.1109/TMECH.2005.852454.

12. Чирков, Д.Г. Методы программной фильтрации данных для работы с ультразвуковыми датчиками в области робототехники / Д.Г. Чирков, А.Д. Стоцкая // Международная конференция по мягким вычислениям и измерениям. - 2020.— Т. 1.—C. 155-158.

13. Матюшкин И.В., Заплетина М.А., Булах Д.А. Введение в геометрическое моделирование: Учеб. пособие. Ч. 1: Формально-математические средства / И.В. Матюшкин, М.А. Заплетина, Д.А. Булах; Министерство образования и науки РФ, Национальный исследовательский университет «МИЭТ».— М.: МИЭТ, 2019. - $240 \mathrm{C}$.

14. Козубовский, В.Р. Бытовые комбинированные приборы двойного назначения: извещатели пожарные газовые и газосигнализаторы загазованности помещений / В.Р. Козубовский, М.В. Федак // Пожаровзрывобезопасность.— 2014.— Т. 23.— № 9.— С. 73-79.

(с Щучкин Евгений Юрьевич ( shchuchkin-pkims@yandex.ru ).

Журнал «Современная наука: актуальные проблемы теории и практики»

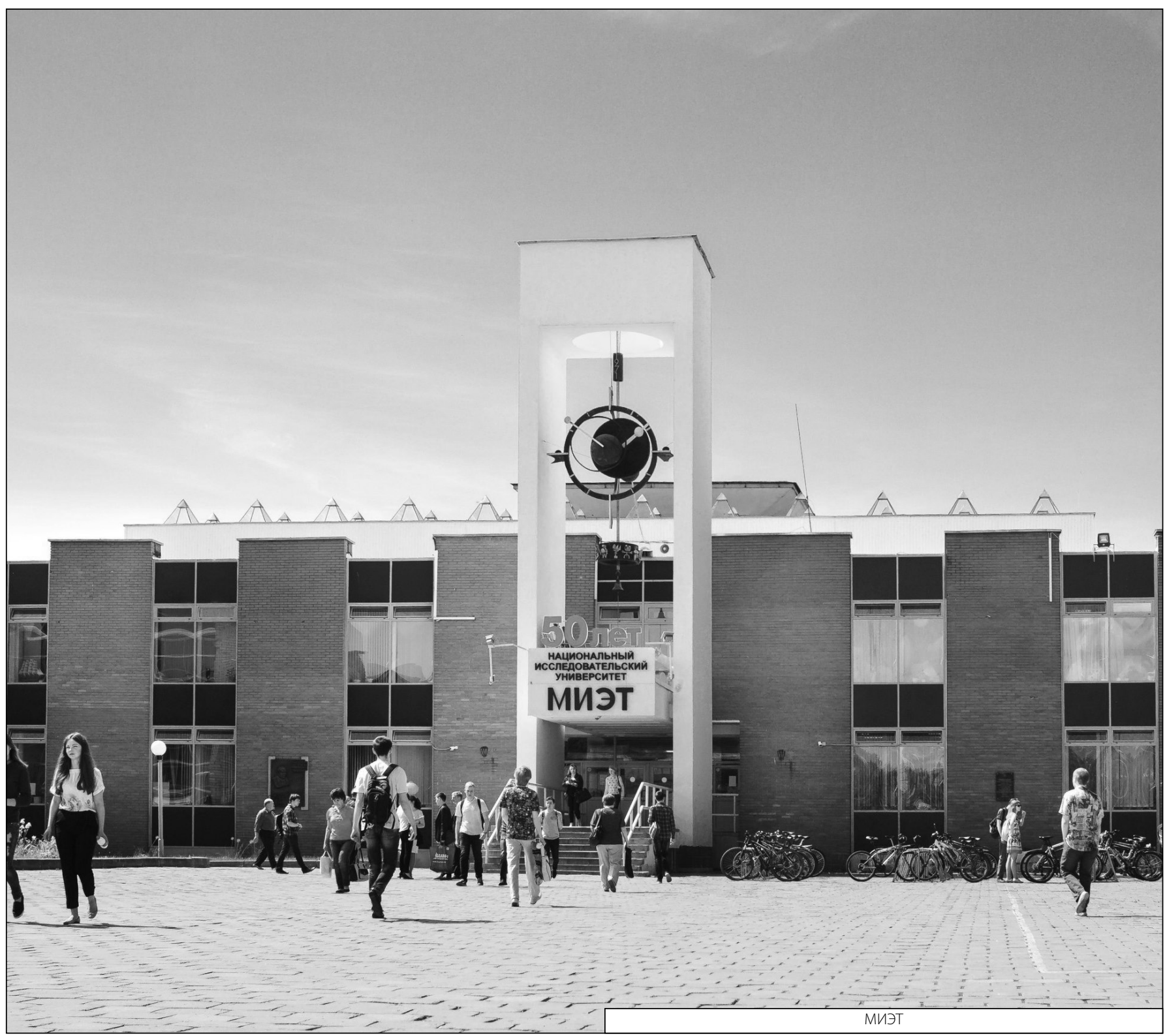

